\title{
CRITICAL FACTORISATION IN SQUARE-FREE WORDS
}

\author{
TERO HARJU* (1)
}

\begin{abstract}
A position $p$ in a word $w$ is critical if the minimal local period at $p$ is equal to the global period of $w$. According to the Critical Factorisation Theorem all words of length at least two have a critical point. We study the number $\eta(w)$ of critical points of square-free ternary words $w$, i.e., words over a three letter alphabet. We show that the sufficiently long square-free words $w$ satisfy $\eta(w) \leq|w|-5$ where $|w|$ denotes the length of $w$. Moreover, the bound $|w|-5$ is reached by infinitely many words. On the other hand, every square-free word $w$ has at least $|w| / 4$ critical points, and there is a sequence of these words closing to this bound.
\end{abstract}

Mathematics Subject Classification. 68R15.

Received August 10, 2021. Accepted January 28, 2022.

\section{INTRODUCTION}

The Critical Factorisation Theorem [2, 4] is one of the gems in combinatorics on words. It states that each word $w$ with $|w| \geq 2$ has a critical point, i.e., a position where the local period $\partial(w, p)$ is equal to the global period $\partial(w)$ of the word. For a word $w$ with a factorisation $w=x y, \partial(w,|x|)$ denotes the length of the shortest word $u$ such that of $u$ and $x$ one is a suffix of the other, and of $u$ and $y$ one is a prefix of the other.

In the binary case, say $w \in\{0,1\}^{*}$, it was shown in [5] that there are words having only one critical point; e.g., the Fibonacci words of length at least five are such. Also, it was shown there that each binary word $w$ of length $|w| \geq 5$ and period $\partial(w)>|w| / 2$ has less than $|w| / 2$ critical points.

We shall now study the number of critical points in ternary square-free words. We show that, each sufficiently long square-free word $w$ can have at most $|w|-5$ critical points, and the bound $|w|-5$ is obtained by infinitely many square-free $w$. Also, we prove that a square-free word $w$ has at least $|w| / 4$ critical points, and that there is a sequence of square-free words closing to this bound.

\section{Preliminaries}

For a more extensive introduction to combinatorics on words, including square-freeness and critical factorisation, we refer to Lothaire [6].

For a finite alphabet $\Sigma$, let $\Sigma^{*}$ denote the monoid of all finite words over $\Sigma$ under concatenation. The empty word is denoted by $\varepsilon$. Let $w \in \Sigma^{*}$. The length $|w|$ of $w$ is the number of the occurrences of its letters. If $w=w_{1} u w_{2}$ then $u$ is a factor of $w$. It is a prefix if $w_{1}=\varepsilon$, and a suffix if $w_{2}=\varepsilon$. The word $w$ is said to be bordered if there exists a nonempty word $v$, with $v \neq w$, that is both a prefix and a suffix of $w$.

Keywords and phrases: Critical point, critical factorisation theorem, ternary words, square-free words.

Department of Mathematics and Statistics, University of Turku, Turku, Finland.

* Corresponding author: harju@utu.fi 


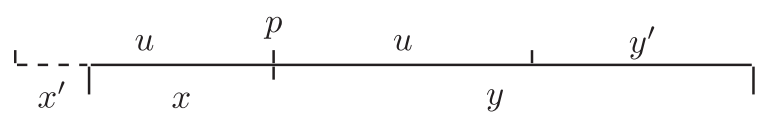

FIgURE 1. A repetition word $u$ of $w=x y$ having left overflow at position $p=|x|$.

A word $w \in \Sigma^{*}$ is square-free if it has no factors of the form $v v$ for nonempty words $v$. Axel Thue [8] showed in 1912 that there are square-free words over a ternary alphabet $\Sigma_{3}=\{0,1,2\}$. One such word is obtained by iterating the following morphism $\tau: \Sigma_{3}^{*} \rightarrow \Sigma_{3}^{*}$ on the initial letter 0 :

$$
\tau(0)=012, \quad \tau(1)=02, \quad \tau(2)=1 .
$$

The iteration ultimately gives an infinite square-free word

$$
\mathbf{m}=012021012102012 \cdots
$$

By the form of the morphism $\tau$, the word $\mathbf{m}$ does not contain the short words 010, 212 and 01201 as its factors. The infinite word $\mathbf{m}$ is sometimes called a variation of Thue-Morse word; see [1].

Lemma 2.1. Let $x$ be a nonempty factor of a square-free word $w$. Then " $x$ does not overlap with itself in $w "$, meaning that if $w=u x_{1} x_{2} x_{3} v$ where $x=x_{1} x_{2}=x_{2} x_{3}$ and $x_{2} \neq \varepsilon$ then $x_{1}=\varepsilon=x_{3}$.

Proof. Overlapping means, see e.g. [6], that $x_{1}$ and $x_{3}$ are conjugates: $x_{1}=r s, x_{3}=s r$ and $x_{2}=(r s)^{k} r$ for some $r, s$ and $k \geq 0$. But $x_{1} x_{2} x_{3}=(r s)^{k+2} r$ does contain a square even if $k=0$.

\section{Critical FACtorisations}

We follow the main notations of [5].

An integer $p$, with $1 \leq p \leq|w|$, is a period of $w$ if for the prefix $u$ of $w$ of length $p, w$ is a prefix of $u^{n}$ for some $n$. The minimal period of $w$ is denoted by $\partial(w)$. We have that $w$ is unbordered if and only if $\partial(w)=|w|$.

An integer $p$ with $1 \leq p<|w|$ is called a position or a point in $w$. It denotes the place after the prefix $x$ of length $p: w=x \cdot y,|x|=p$. Thus there are $|w|-1$ positions in $w$. A nonempty word $u$ is a repetition word of $w$ at $p$ if there are words $x^{\prime}$ and $y^{\prime}$ (possibly empty) such that $u=x^{\prime} x$ or $x=x^{\prime} u$, and $u=y y^{\prime}$ or $y=u y^{\prime}$. If here $|u|>|x|$ (resp. $|u|>|y|$ ) then $u$ is said to have left overflow (resp., right overflow) at $p$; see Figure 1.

The length of a repetition word of $w$ at $p$ is called a local period at $p$. The minimal local period of $w$ at $p$ is denoted by

$$
\partial(w, p)=\min \{q \mid q \text { a local period of } w \text { at } p\}
$$

Clearly, the (global) period $\partial(w)$ is a local period at every point, and hence $\partial(w, p) \leq \partial(w)$ for all $p$. A position $p$ of $w$ is said to be critical if $\partial(w, p)=\partial(w)$.

The following result follows from the minimality assumption on $\partial(w, p)$.

Lemma 3.1. A repetition word $u$ of $w$ at $p$ of length $\partial(w, p)$ is unique and it is unbordered.

For a word $w$, we let

$$
\eta(w)=\text { the number of critical points of } w \text {. }
$$




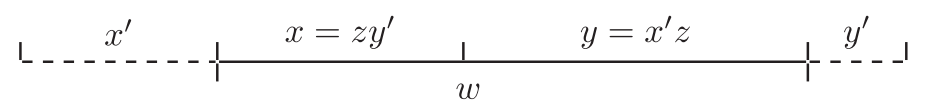

FIgURE 2. Left and right overflows imply criticality.

The number

$$
\frac{\eta(w)}{|w|-1}
$$

is called the density of the critical points in $w$.

Example 3.2. Let $w=0120201202021021021$ be an unbordered word of length 19, i.e., $\partial(w)=|w|$. It is not square-free. The minimal local periods of $w$ are in order of the 18 positions

$$
3,5,5,2,5,5,19,19,2,2,19,19,3,3,3,3,3,3 .
$$

In this example, $\eta(w)=4$, and the density of critical points is $4 / 18=0.222 \ldots$

The Critical Factorisation Theorem is due to Césari and Vincent [2]. The present form of the theorem was developed by Duval [4]; for the proofs, see also [3], [5] and Chapter 8 in [7].

Theorem 3.3 (Critical Factorisation Theorem). Every word $w$ of length $|w| \geq 2$ has a critical point. Moreover, there is a critical point $p$ satisfying $p \leq \partial(w)$.

Lemma 3.4. Let $u$ be a repetition word of $w$ at $p$ with $|w| \geq 2$ of length $\partial(w, p)$. If $u$ has both left and right overflows at $p$ then $p$ is a critical point.

Proof. Let $w=x y$ where $u=x^{\prime} x=y y^{\prime}$ for nonempty words $x^{\prime}, y^{\prime}$; see Figure 2. By symmetry, we may assume that $\left|x^{\prime}\right| \leq|y|$ (otherwise $\left|y^{\prime}\right| \leq|x|$ ). Therefore $y=x^{\prime} z$ and $x=z y^{\prime}$ for some $z$. Now, $w=x y=z y^{\prime} x^{\prime} z$, and hence $\left|z y^{\prime} x^{\prime}\right|$ is a period of $w$, i.e., $\partial(w) \leq\left|z y^{\prime} x^{\prime}\right|$. But $\left|z y^{\prime} x^{\prime}\right|=\left|x^{\prime} z y^{\prime}\right|=|u|$ which shows that $\partial(w, p)=|u|=\partial(w)$ implying that $p$ is a critical point.

\section{MAXIMUM NUMBER OF CRITICAL POINTS}

We notice first that if $w$ is a square-free word with $|w| \geq 2$, then $\partial(w, p) \geq 2$ for all positions $p$, since $\partial(w, p)=1$ would imply a factor of the form $a a$ in $w$.

The next lemma follows from the observation that if a point $p$ of $w$ has neither left nor right overflow, the minimal repetition word $u$ at $p$ supplies a square $u u$ in $w$.

Lemma 4.1. A word $w$ with $|w| \geq 2$ is square-free if and only if each repetition word at each position $p$ has left or right overflow, or both.

Example 4.2. The square-free word $w=01020120210201021$ of length 17 is unbordered, i.e., $\partial(w)=17$. It has 9 critical points at the consecutive positions $p=5,6, \ldots, 13$. This gives the density number $9 / 16 \approx 0.56$. For instance, the position $p=4$ has the minimal repetition word $u=012021020102$, since $u$ is the shortest factor after the prefix 0102 that ends with 0102 . Thus $\partial(w, 4)=12$.

For a word $w$, let

$$
M(w)=\left\lfloor\frac{|w|+1}{2}\right\rfloor
$$

denote the midpoint of $w$. For odd length $|w|$, it is just a choice of the two points nearest to the centre of $w$. 
Lemma 4.3. For a square-free word $w \in \Sigma_{3}^{*}$, the position $M(w)$ is critical.

Proof. For even $|w|$, the claim is clear from Lemma 4.1.

Suppose then that $|w|=2 k+1$, and let $u$ be the minimal local repetition word of $w$ at $M(w)=k+1$. Suppose $u$ has right but not left overflow. Then $|u|=k+1$, and hence $w=v a v$ where $u=v a$ for a prefix $v$ and an overflow letter $a$. But then $\partial(w, k+1)=|u|=\partial(w)$, and the claim follows.

Theorem 4.4. The minimal local periods form a unimodular sequence for square-free ternary words $w \in \Sigma_{3}^{*}$ with $|w| \geq 2$, i.e.,

$$
\begin{array}{lll}
\partial(w, p-1) \leq \partial(w, p) & \text { for } & 2 \leq p \leq M(w) \\
\partial(w, p) \leq \partial(w, p-1) & \text { for } & p-1 \geq M(w)
\end{array}
$$

In particular, the critical points $p$ of $w$ form an interval $q_{0} \leq p \leq q_{1}$ for some $q_{1} \leq M(w)$ and $q_{2} \geq M(w)$.

Proof. Let $2 \leq p \leq M(w)$. The cases for $p \geq M(w)$ follow by considering the reverse of the word $w$ which is also square-free. Let the minimal repetition word of $w$ at $p$ be $u$, i.e., $|u|=\partial(w, p)$. Since $w$ is square-free and $|u| \geq 2, u$ has left overflow. If it also has right overflow then $p$ is critical by Lemma 3.4. Let $a$ be the letter such that $u=v a$. Then $|a v|$ is a local period at $p-1$ since the position $p-1$ has a repetition word $a v$. (It need not be minimal.) Hence $\partial(w, p-1) \leq \partial(w, p)$.

For the second claim, by Lemma 4.3, $w$ has a critical point $p$ with $p \leq M(n)$ and a critical point $q \geq M(w)$. This proves the claim.

Example 4.5. Consider the prefix $w=\tau^{5}(0)$ of the square-free word $\mathbf{m}$, i.e.,

$$
w=012021012102012021020121 .
$$

It is unbordered with $\mid w[=24$. The sequence of the 23 minimal local periods is

$$
3,6,6,12,12,12,12,24, \ldots, 24,14,14,6,2 \text {. }
$$

Thus $\eta(w)=12$, i.e., just over one half of the positions are critical.

Theorem 4.6. For each square-free ternary word $w$ of length $|w| \geq 26$, we have $\eta(w) \leq|w|-5$.

Proof. Let $w \in \Sigma_{3}^{*}$ be a square-free ternary word of length $n \geq 26$. We show that $w$ has at least four non-critical points among the $n-1$ positions. The points 1 and $n-1$ are always non-critical, since every letter of $\Sigma_{3}$ occurs in every factor of length four. Let us then assume that $w$ has exactly three non-critical points. Therefore at least one of the positions 2 or $n-2$ is critical. Without restriction, we can say that $p=2$ is critical.

Without restriction we may assume that 01 is a prefix of $w$. It can be checked that there are no such squarefree words of length 15 where 01 occurs only as a prefix. After inspection, we find that the only such word of length 14 is $v=01210212021020$.

Thus since $|w| \geq 15$, we have $w=01 x 01 y$ for some words $x$ and $y$ such that 01 does not occur in $x$. Now, the word $x 01$ is a repetition of $w$ at position 2 and hence, by the criticality of $p=2$, we have $|x 01|=\partial(w)$. Because $w$ is square-free, $y$ must be a proper prefix of $x$. The word $x$ does not have any occurrences of 01 and it cannot end in the letter 0 . Thus $|01 x| \leq 13$. But now $n \leq 25$; a contradiction.

Example 4.7. In contrast to Theorem 4.6, the word $w=01210212021020121021202$ of length 23 with $\partial(w)=13$ has only three non-critical points, $p=1,2,22$.

The upper bound on the critical points is optimal:

Theorem 4.8. There are arbitrarily long square-free words $w \in \Sigma_{3}^{*}$ with $\eta(w)=|w|-5$. 
TABLE 1. Local periods of non-critical points.

\begin{tabular}{ccc}
\hline$p$ & $\partial(w, p)$ & Rep. word \\
\hline 1 & 2 & 10 \\
2 & 4 & 0201 \\
$|w|-2$ & 4 & 1202 \\
$|w|-1$ & 2 & 21 \\
\hline
\end{tabular}

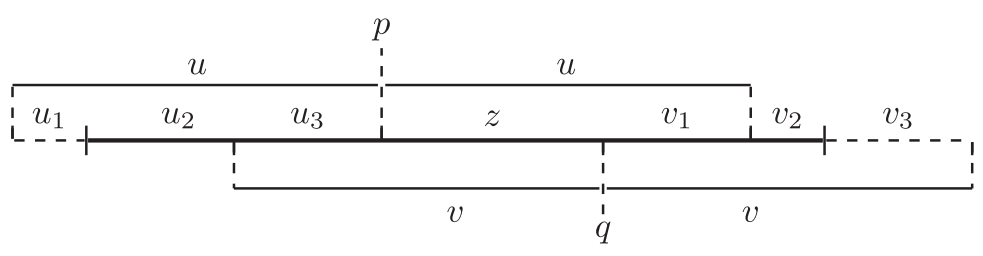

FIgURE 3 . The repetition words $u$ and $v$ for non-critical points $p$ and $q$.

Proof. We rely on the infinite square-free word $\mathbf{m}$ that is a fixed point of the morphism $\tau$. Consider the factors of $\mathbf{m}$ of the form $\beta=10201 \alpha 12021$. For our purpose, it suffices to choose the words $\beta$ that start after the position 9 of $\mathbf{m}$, i.e., just after the prefix 012021012. There are infinitely many words $\beta$ since the suffix 12021 is a factor of $\tau^{2}(0)$.

For fixed middle word $\alpha$, consider $w=0 \beta 2=010201 \alpha 120212$ that begins and ends in the 'forbidden' words 010 and 212 that do not occur in $\mathbf{m}$. It is, clearly, square-free and unbordered. Each point $p$ with $2<p<|w|-2$ is critical, since the minimal repetition word at $p$ must have both left and right overflow in order to leap over a factor 010 or 212; see Lemma 3.4. Table 1 lists the local periods and the minimal repetition words for the remaining four (non-critical) points.

\section{Minimum NUMBER OF CRITICAL POINTS}

We now turn to the minimality problem of critical points in square-free words.

Theorem 5.1. For each square-free word $w \in \Sigma_{3}^{*}$ with $|w| \geq 2$, we have $\eta(w) \geq|w| / 4$.

Proof. Let $w \in \Sigma_{3}^{*}$ be a square-free word of length $|w|=n$. We remind that, by Lemma 4.3, the middle point $M(w)$ is always critical in $w$. We show that the distance between two non-critical points on the opposite sides of the middle point is at least $n / 4$. The claim then follows from Theorem 4.4.

Assume, contrary to the claim, that $p$ and $q$ are non-critical points such that

$$
p<n / 2<q \text { and } q-p<n / 4 .
$$

Let $u$ and $v$ be the minimal repetition words at $p$ and $q$, respectively. Consequently, the word $u$ has left overflow, and $v$ has right overflow. Observe that $p>n / 4$ and $q<3 n / 4$. From $p>n / 4$ it follows that $|u|>n / 4$. Similarly $|v|>n / 4$ and $q-|v|<n / 2$. Since $q-p<n / 4$, we have $p+|u| \geq q$, i.e., the second occurrence of $u$ reaches over the position $q$. Similarly the first occurrence of $v$ starts before the position $p$; see Figure 3 , where $|z|=q-p$.

We now rely on the notations of the factors in Figure 3.

The words $u_{3}$ and $v_{1}$ are both prefixes of $v$ and suffixes of $u$. If $\left|v_{1}\right|>\left|u_{3}\right|$ then, as prefixes of $v$, we have $v_{1}=u_{3} x$ for some nonempty $x$. But then $x$ is a border of $u$ since $u_{3}$ cannot overlap with itself at the end of the first occurrence of $u$; a contradiction. Similarly, if $\left|u_{3}\right|>\left|v_{1}\right|$ then, as suffixes of $u$, we have $u_{3}=x v_{1}$ for some 
nonempty $x$ yielding that $x$ is is a border of $v$; a contradiction. Therefore $v_{1}=u_{3}$. In this case $z=u_{1} u_{2}=v_{2} v_{3}$, and

$$
w=u_{2} v_{1} z v_{1} v_{2}=u_{2} v_{1} u_{1} u_{2} v_{1} v_{2} .
$$

Since $u_{1} u_{2}=v_{2} v_{3}$, one of $u_{1}$ or $v_{2}$ is a prefix of the other. To avoid $\left(u_{2} v_{1} u_{1}\right)^{2}$ in $w$, the word $v_{2}$ must be a proper prefix of $u_{1}$. But now $\partial(w) \leq\left|u_{2} v_{1} u_{1}\right|=|u|=\partial(w, p)$ contradicting the assumption that $p$ was not critical. This proves the claim.

For the existence part of the next theorem, we take a quick technical analysis of the prefixes of the word $\mathbf{m}$. An induction argument gives $\left|\tau^{n}(0)\right|=3 \cdot 2^{n-1},\left|\tau^{n}(1)\right|=2^{n}$ and $\left|\tau^{n}(2)\right|=2^{n-1}$. For instance,

$$
\left|\tau^{n+1}(0)\right|=\left|\tau^{n}(012)\right|=3 \cdot 2^{n-1}+2^{n}+2^{n-1}=3 \cdot 2^{n} .
$$

Define the words $\mathbf{m}_{n}$, for $n \geq 1$, as follows

$$
\mathbf{m}_{n}=\tau^{2 n-1}(0) \tau^{2 n-3}(0) \cdots \tau^{3}(0) \tau(0) .
$$

We show that $\mathbf{m}_{n} 0$ is a prefix of $\mathbf{m}$ of length $4^{n}$. First $\mathbf{m}_{1} 0=0120=\tau(0) 0$ is a prefix of $\mathbf{m}$. Inductively, we have

$$
\tau^{2}\left(\mathbf{m}_{n} 0\right)=\tau^{2 n+1}(0) \tau^{2 n-1}(0) \cdots \tau^{3}(0) \tau^{2}(0)=\mathbf{m}_{n+1} 0 \cdot 21 .
$$

and hence also $\mathbf{m}_{n+1} 0$ is a prefix of $\mathbf{m}$.

For the length of $\mathbf{m}_{n}$, we obtain

$$
\left|\mathbf{m}_{n}\right|=\sum_{i=1}^{n} 3 \cdot 2^{2(n-i)}=3 \sum_{i=1}^{n} 4^{n-i}=4^{n}-1 .
$$

As a prefix of $\mathbf{m}$, the word $\mathbf{m}_{n}$ is square-free.

Theorem 5.2. For all real numbers $\delta>0$, there exists a square-free ternary word $w=w(\delta)$ the density of which satisfies

$$
0.25<\frac{\eta(w)}{|w|}<0.25+\delta .
$$

Proof. For any square-free word $x \in \Sigma_{3}^{*}$, let

$$
w_{x}=0 x 02 x 10 x 02 x 0 .
$$

Suppose first that $w_{x}$ is square-free, and thus that $x$ does not overlap with itself in $w_{x}$. The suffix $2 x 0$ of $w_{x}$ does not occur elsewhere in $w_{x}$, and hence the point $3|x|+6$ is critical, since it must have both overflows. It is the rightmost critical point. Indeed, $\partial\left(w_{x}, 3|x|+7\right)=|x|+2$. For the point $2|x|+3$, the minimal repetition word is $10 x 02 x$ of length $2|x|+4<\partial(w)$ since $\partial(w)>3|x|+7$. By Lemma 4.3, the middle point is critical, and hence the position $2|x|+4$ is the leftmost critical point. It follows that $w_{x}$ has $(3|x|+7)-(2|x|+4)=|x|+3$ critical points. Thus

$$
\frac{\eta(w)}{\left|w_{x}\right|}=\frac{|x|+3}{4|x|+8}=0.25+\frac{1}{\left|w_{x}\right|}
$$


which has the limit 0.25 as $|x| \rightarrow \infty$.

It remains to show that there are arbitrarily long square-free words $x$ for which $w_{x}$ is square-free. Again, we lean on the word $\mathbf{m}$. We consider the words $w_{x_{n}}$ where

$$
x_{n}=120102 \mathbf{m}_{n} .
$$

We have

$$
w_{x_{n}}=0 \cdot 120102 \mathbf{m}_{n} \cdot 02 \cdot 120102 \mathbf{m}_{n} \cdot 10 \cdot 120102 \mathbf{m}_{n} \cdot 02 \cdot 120102 \mathbf{m}_{n} \cdot 0
$$

Since 010 and 212 do not occur in $\mathbf{m}$, both 010 and 212 would have to be aligned in any square $u u$ of $w_{x_{n}}$, which is not possible by the 'markers' 02,1 and 0 dividing the word. Also, since $\mathbf{m}_{n}$ has a border $\tau(0)$, one easily checks that there are no short squares $u u$ in $w_{x}$ for $|u| \leq 4$. Hence a possible square must be inside one of the words (a) $102 \mathbf{m}_{n} 021$, (b) $102 \mathbf{m}_{n} 101201$, or (c) $102 \mathbf{m}_{n} 0$. We consider these cases separately. Recall that $\mathbf{m}_{1}=012=\tau(0)$. Also, since $\mathbf{m}$ is a fixed point of the morphism $\tau$, whenever $v$ is a factor of $\mathbf{m}$, so is $\tau(v)$.

(a) Let $\alpha_{n}=102 \mathbf{m}_{n} 021$. The word $\alpha_{1}=102012021$ occurs in $\mathbf{m}$ after position 9 . We prove by induction that each $\alpha_{n}$ is a factor of $\mathbf{m}$, and thus they are square-free. Suppose, using (5.2), that

$$
\alpha_{i}=102 \mathbf{m}_{i} 021=102 \tau^{2 i-1}(0) \cdots \tau(0) 021
$$

is a factor of $\mathbf{m}$. Then

$$
\tau\left(\alpha_{i}\right)=0201 \cdot 21 \tau^{2 i}(0) \cdots \tau^{2}(0) 012 \cdot 021
$$

where the indicated factor will be denoted by $z=21 \tau^{2 i}(0) \cdots 012$. By mapping with $\tau$, we obtain

$$
\tau(z)=102 \tau^{2 i+1}(0) \cdots \tau^{3}(0) \tau(0) 021=102 \mathbf{m}_{i+1} 021=\alpha_{i+1} .
$$

Hence $\alpha_{n}$ is a factor of $\mathbf{m}$ for all $n$.

(b) We employ in this case the same techniques as in (a) except that we need to eliminate the last letter 1 of the word. In order for $102 \mathbf{m}_{n} 101201$ to have a square $u u$, the former occurrence of $u$ in the square must be a factor of $102 \mathbf{m}$. However, $\mathbf{m}$ does not have a factor 01201 since it would have to be part of the square 012012. Therefore we can, and must, choose $\beta_{n}=102 \mathbf{m}_{n} 101202$.

The first occurrence of $\beta_{1}=102 \mathbf{m}_{1} 101202=102 \tau(0) 101202$ in $\mathbf{m}$ starts after position 17 . We proceed inductively as in case (a). Suppose that

$$
\beta_{i}=102 \mathbf{m}_{i} 101202=102 \tau^{2 i-1}(0) \cdots \tau(0) 101202
$$

is a factor of $\mathbf{m}$. Mapping by $\tau$ gives

$$
\tau\left(\beta_{i}\right)=0201 \cdot 21 \tau^{2 i}(0) \cdots \tau^{2}(0) 0201 \cdot 20210121
$$

where the indicated portion $z=21 \tau^{2 i}(0) \cdots \tau^{2}(0) 0201$ gives

$$
\tau(z)=102 \tau^{2 i+1}(0) \cdots \tau^{3}(0) \tau(0) 101202=102 \mathbf{m}_{i+1} 101202=\beta_{i+1} .
$$

Hence $\beta_{n}$ is a factor of $\mathbf{m}$, and thus square-free, for all $n$.

(c) The word $102 \mathbf{m}_{n} 0$ is a factor of $\alpha_{n}$ and thus square-free. This proves the claim. 
The chosen words $x_{n}=120102 \mathbf{m}_{n}$ are not the only ones that give a square-free word $w_{x}$.

Problem 5.3. Does there exist, for all sufficiently large $n$, a word $x$ of length $n$ such that $w_{x}$ is square-free?

Problem 5.4. Does there exist a word $w$ such that $\eta(w)=|w| / 4$ ?

Acknowledgements. The author thanks for the kind referees for their comments that clairifed the proofs of the article.

\section{REFERENCES}

[1] F. Blanchet-Sadri, J.D. Currie, N. Rampersad and N. Fox, Abelian complexity of fixed point of morphism $0 \mapsto 012,1 \mapsto 02,2 \mapsto$ 1. Integers 14 (2014) a11.

[2] Y. Césari and M. Vincent, Une caractérisation des mots périodiques. C. R. Acad. Sci. Paris 286(A) (1978) $1175-1177$.

[3] M. Crochemore and D. Perrin, Two-way string-matching. J. Assoc. Comput. Mach. 38 (1991) 651-675.

[4] J.-P. Duval, Périodes et répétitions des mots du monoïde libre. Theoret. Comput. Sci. 9 (1979) 17-26.

[5] T. Harju and D. Nowotka, Density of critical factorizations. Theor. Inform. Appl. 36 (2002) 315-327.

[6] M. Lothaire, Combinatorics on words. Vol. 17 of Encyclopedia of Mathematics. Addison-Wesley, Reading, Massachusetts (1983).

[7] M. Lothaire, Algebraic Combinatorics on Words. Cambridge University Press, Cambridge, United Kingdom (2002).

[8] A. Thue, Über die gegenseitige Lage gleicher Teile gewisser Zeichenreihen. Norske Vid. Selsk. Skr., I Mat.-nat. Kl. Christiania 1 (1912) 1-67.

\section{Subscribe to Open (S2O) A fair and sustainable open access model}

This journal is currently published in open access under a Subscribe-to-Open model (S2O). S2O is a transformative model that aims to move subscription journals to open access. Open access is the free, immediate, online availability of research articles combined with the rights to use these articles fully in the digital environment. We are thankful to our subscribers and sponsors for making it possible to publish this journal in open access, free of charge for authors.

\section{Please help to maintain this journal in open access!}

Check that your library subscribes to the journal, or make a personal donation to the S2O programme, by contacting subscribers@edpsciences.org

More information, including a list of sponsors and a financial transparency report, available at: https://www.edpsciences.org/en/maths-s2o-programme 